\title{
Sylvie FAINZANG
}

Anthropologue, spécialisée dans le domaine de la santé.

Directeur de recherche à l'Inserm et membre du CERMES

Centre de Recherche Médecine, Sciences, Santé et Société)

(2000)

\section{"La maladie, un objet pour l'anthropologie sociale”}

Un document produit en version numérique par Jean-Marie Tremblay, bénévole, professeur de sociologie au Cégep de Chicoutimi

Courriel: jean-marie tremblay@uqac.ca

Site web pédagogique : http://www.uqac.ca/jmt-sociologue/

Dans le cadre de: "Les classiques des sciences sociales" Une bibliothèque numérique fondée et dirigée par Jean-Marie Tremblay, professeur de sociologie au Cégep de Chicoutimi Site web: http://classiques.uqac.ca/

Une collection développée en collaboration avec la Bibliothèque Paul-Émile-Boulet de l'Université du Québec à Chicoutimi Site web: http://bibliotheque.uqac.ca/ 


\section{Politique d'utilisation de la bibliothèque des Classiques}

Toute reproduction et rediffusion de nos fichiers est interdite, même avec la mention de leur provenance, sans l'autorisation formelle, écrite, du fondateur des Classiques des sciences sociales, Jean-Marie Tremblay, sociologue.

Les fichiers des Classiques des sciences sociales ne peuvent sans autorisation formelle:

- être hébergés (en fichier ou page web, en totalité ou en partie) sur un serveur autre que celui des Classiques.

- servir de base de travail à un autre fichier modifié ensuite par tout autre moyen (couleur, police, mise en page, extraits, support, etc...),

Les fichiers (.html, .doc, .pdf., .rtf, .jpg, .gif) disponibles sur le site Les Classiques des sciences sociales sont la propriété des Classiques des sciences sociales, un organisme à but non lucratif composé exclusivement de bénévoles.

Ils sont disponibles pour une utilisation intellectuelle et personnelle et, en aucun cas, commerciale. Toute utilisation à des fins commerciales des fichiers sur ce site est strictement interdite et toute rediffusion est également strictement interdite.

L'accès à notre travail est libre et gratuit à tous les utilisateurs. C'est notre mission.

Jean-Marie Tremblay, sociologue

Fondateur et Président-directeur général, LES CLASSIQUES DES SCIENCES SOCIALES. 
Cette édition électronique a été réalisée par Jean-Marie Tremblay, bénévole, professeur de sociologie au Cégep de Chicoutimi à partir de :

Sylvie FAINZANG

“La maladie, un objet pour l’anthropologie sociale”.

Un article publié dans la revue Ethnologie comparées, Revue électronique semestrielle, no 1, 2000. Université de Montpellier 3, France.

[Autorisation formelle accordée par l'auteure le 11 février 2009 de diffuser cette œuvre dans Les Classiques des sciences sociales.]

Courriel : sylvie.fainzang@orange.fr

Polices de caractères utilisée :

Pour le texte: Times New Roman, 12 points.

Pour les citations : Times New Roman, 12 points.

Pour les notes de bas de page : Times New Roman, 12 points.

Édition électronique réalisée avec le traitement de textes Microsoft Word 2008 pour Macintosh.

Mise en page sur papier format : LETTRE (US letter), 8.5’’ x 11’’)

Édition numérique réalisée le 14 février 2009 à Chicoutimi, Ville de Saguenay, province de Québec, Canada.

\section{Fait avec}

Macintosh 


\section{Sylvie FAINZANG}

Anthropologue, spécialisée dans le domaine de la santé.

Directeur de recherche à l'Inserm et membre du CERMES

Centre de Recherche Médecine, Sciences, Santé et Société)

\section{"La maladie, un objet pour l'anthropologie sociale”}

\section{Ethnologies comparées}

Revue électronique semestrielle

http://alor.univ-montp3.fr/cerce/revue.htm

Un article publié dans la revue Ethnologie comparées, Revue électronique semestrielle, no 1, 2000. Université de Montpellier 3, France. 


\section{Table des matières}

$\underline{\text { Introduction }}$

Naissance de l'anthropologie médicale

Les grandes orientations de la recherche

L'orientation fonctionnaliste

$\underline{\text { L'orientation cognitive }}$

De l'anthropologie médicale à l'anthropologie de la maladie

Remarques épistémologiques

$\underline{\text { Références bibliographiques }}$ 
Sylvie FAINZANG

"La maladie, un objet pour l'anthropologie sociale".

Un article publié dans la revue Ethnologie comparées, Revue électronique semestrielle, no 1, 2000. Université de Montpellier 3, France.

\section{INTRODUCTION}

\section{$\underline{\text { Retour à la table des matières }}$}

Pour tenter de cerner ce qu'est ce nouveau savoir constitué par l'anthropologie médicale, il faut d'abord dissiper un malentendu. Ce malentendu, c'est celui qui consiste à envisager cette discipline comme une branche des sciences médicales qui porterait son attention sur les conceptions culturelles du mal, en vue d'aider, dans leur tâche, les professionnels de la santé. Un tel malentendu aboutit à situer l'anthropologie médicale en marge de ce qui la définit comme anthropologie sociale et culturelle et empêche de comprendre en quoi l'approche de la maladie constitue, pour l'anthropologue, un objet de connaissance comme un autre.

On entend généralement comme relevant du domaine de l'anthropologie médicale les travaux qui portent leur attention sur les représentations de la maladie, les itinéraires des malades, le rôle des thérapeutes ou les pratiques thérapeutiques de toutes sortes (dont les rituels de guérison), en fonction du système socioculturel dans lequel ils s'insèrent. Je me propose ici de présenter la genèse et l'évolution de ce domaine de recherche, et quelques exemples de ses problématiques et de ses objets.

À ce propos, j'expliciterai la différence entre "anthropologie médicale" (dénomination ambiguë puisqu'on ne distingue pas clairement s'il s'agit d'une branche 
de l'anthropologie ou d'une branche de la médecine) et "anthropologie de la maladie" (formulation proposée par Marc Augé) pour faire ressortir les implications théoriques de ces deux intitulés, et leurs finalités respectives.

\section{Naissance de l'anthropologie médicale}

$\underline{\text { Retour à la table des matières }}$

L'anthropologie médicale repose sur le postulat que la Maladie (fait universel) est gérée et traitée suivant des modalités différentes selon les sociétés et que ces modalités sont liées à des systèmes de croyances et de représentations déterminés, en fonction de la culture dans laquelle elle émerge. Bien que cet énoncé n'ait été systématisé que plus tard, on en trouve l'ébauche chez Rivers (1924) dont les travaux sur la médecine magico-religieuse lui valent d'être considéré comme un précurseur de la discipline.

L'objet principal des travaux de Rivers a été de cerner la nature des concepts de maladie élaborés par les différentes sociétés. Il a tenté le premier de relier de façon systématique les médecines traditionnelles et d'autres aspects de la culture et de l'organisation sociale. Dans Medicine, Magic and Religion, Rivers montre que les pratiques médicales des sociétés "primitives" découlent de certaines croyances médicales, et qu'elles prennent un sens en fonction de ces croyances. Il a ainsi suggéré d'une part que la pratique médicale était un processus social qu'il fallait étudier au même titre que les autres processus sociaux, et que, d'autre part, les conceptions médicales qui sont selon nous erronées, n'en sont pas moins fondées sur un système de croyances cohérent et logique.

A la suite de Rivers, un certain nombre d'auteurs ont contribué à constituer l'anthropologie médicale en un domaine à part entière, relevant de l'anthropologie sociale et culturelle. Les principaux travaux effectués en anthropologie médicale ont été marqués par deux grandes orientations : l'orientation fonctionnaliste et l'orientation cognitive. 


\section{Les grandes orientations de la recherche}

\section{L'orientation fonctionnaliste}

\section{$\underline{\text { Retour à la table des matières }}$}

On peut ranger sous cette catégorie les travaux dont l'objectif principal a été de rechercher la fonction sociale des représentations de la maladie dans les sociétés étudiées. Dans un article fort perspicace écrit dès 1941, Hallowell montre que l'interprétation et le traitement de la maladie remplissent une fonction de contrôle social dans les sociétés dépourvues d'institutions politiques et judiciaires spécialisées pour régler les conflits et imposer le respect de leurs normes.

Quelque temps plus tard, et dans la même orientation, Ackerknecht (1946) renouvelle ce type de problématique, et s'inspire du relativisme culturel pour réaffirmer l'unicité de chaque culture et par conséquent, selon lui, de chaque modèle médical. L'essentiel de la thèse de E. Ackerknecht est que les différentes parties constitutives d'un modèle médical sont liées entre elles de manière fonctionnelle. Suivant la voie ouverte par Hallowell, Ackerknecht souligne donc à son tour la fonction de contrôle social jouée par la conceptualisation et le traitement de la maladie dans les sociétés traditionnelles. Il écrit notamment, de façon assez saisissante : "Dans la société primitive, la médecine magico-religieuse fournit, aux moindres frais, les services qui sont rendus dans la nôtre par les Tribunaux, la police, les maîtres d'école, les prêtres ou les soldats" (1971 : 168).

Cette thèse est également servie par les travaux de Turner (1968) qui illustre, avec l'exemple des Ndembu de Zambie, le rôle social que remplit l'institution divinatoire, chargée d'élaborer le diagnostic de la maladie (révéler les causes du malheur, de la maladie ou de la mort). En l'occurrence, l'ethnographie des rituels ndembu révèle que pour déterminer les causes de la maladie, le devin enquête sur le contexte social particulier du malade, procédant ainsi à une véritable analyse de la situation sociale du groupe (des positions que chacun y occupe, et des relations qu'il entretient avec son entourage). Pour Turner également, donc, la consultation divinatoire, à travers sa fonction diagnostique, a certaines affinités avec la procé- 
dure judiciaire, au cours de laquelle sont révélés les conflits sous-jacents en vue d'y remédier. Il s'agit donc, avec la divination, de réparer les perturbations du champ social.

Parvenus au postulat que la médecine est un art des usages sociaux de la maladie, de nombreux anthropologues, notamment anglo-saxons, se sont donc attachés à identifier les mécanismes par lesquels une société assure un contrôle social sur les individus, et cela, au moyen de la maladie, de ses représentations et des pratiques qui lui sont associées. Ces recherches ont permis de montrer que la maladie, en tant qu'événement malheureux affectant à la fois l'individu et le groupe, est génératrice de pratiques qui débordent le champ strictement médical.

Toutefois, bien que leurs analyses conservent en partie leur validité pour de nombreuses sociétés, ces recherches ont en partie occulté les conduites de résistance que les individus peuvent développer à l'égard de ce contrôle social. On le constate par exemple chez les Bisa du Burkina Faso où l'apparition de la maladie est souvent interprétée comme la sanction, infligée par les ancêtres ou les puissances surnaturelles, d'une conduite réprouvée par la société, telle que le vol, l'adultère, le manque de respect à un vieux (nous sommes là dans le contexte d'une organisation sociale basée sur l'opposition entre aînés et cadets), la négligence à accomplir son devoir, etc. Le traitement de la maladie passera par la réparation du mal commis, considéré à l'origine de la maladie. Cependant, les catégories explicatives de la maladie peuvent aussi être manipulées par les individus de façon à tenter de se soustraire au contrôle assumé par l'institution divinatoire ( $c f$. Fainzang 1986).

\section{L'orientation cognitive}

$\underline{\text { Retour à la table des matières }}$

Cette orientation se focalise sur les manières dont les différentes cultures perçoivent et structurent l'expérience. Elle cherche à identifier les catégories forgées par ces cultures pour comprendre l'expérience de la maladie.

Les travaux d'Evans-Pritchard (1968) sur les conceptions sorcellaires des Azandé ont montré que les croyances (en apparence irrationnelles) relatives à 
l'étiologie et à la résolution du malheur, prenaient sens dans leur contexte. EvansPritchard est désormais devenue une référence obligée quand on veut signifier que, dans les sociétés dites traditionnelles, l'apparition du malheur s'intègre dans un dispositif explicatif qui renvoie à l'ensemble des représentations sociales du groupe. Evans-Pritchard illustre cet énoncé par l'exemple célèbre de la chute du toit d'un grenier. Un grenier est une construction à base de terre et de matières végétales, où sont entreposées les récoltes (en l'occurrence des céréales) et dont le toit avancé, produit de l'ombre. Nous sommes donc devant une situation banale de la vie quotidienne : celle où un homme est assis et se repose à l'ombre du toit d'un grenier. Imaginons que le toit tombe sur cet homme, celui-ci va reconnaître deux séries causales indépendantes : $1^{\circ}$ ) certes, le grenier s'est effondré parce qu'il était mal fixé ou que les bois de soutènement en étaient défectueux ; $2^{\circ}$ ) mais c'est sur telle personne et à tel moment qu'il est tombé ; et il y a là, pour les Zandé, un signe à décrypter (par exemple, c'est le signe que l'homme en question a commis une faute ou qu'il est victime d'une persécution par un tiers).

Avec Evans-Pritchard, la nécessité est soulignée avec une plus grande acuité d'envisager l'objet maladie et le système médical d'une société donnée, en relation avec la totalité socioculturelle de celle-ci, et de rechercher les liens existant entre les différents aspects de la vie du groupe (organisation sociale, mode de vie, croyances, rituels, etc.).

\section{De l'anthropologie médicale à l'anthropologie de la maladie}

$\underline{\text { Retour à la table des matières }}$

Par la suite, les recherches menées en anthropologie médicale recèlent deux attitudes inverses mais non exclusives, propres à infléchir l'orientation des auteurs, et que l'on peut résumer de la manière suivante :

1. l'examen des problèmes relatifs à la santé et à la maladie considérés dans une perspective anthropologique, peut contribuer à enrichir la recherche médicale ; 
2. les problèmes posés dans le domaine de l'anthropologie sociale et culturelle trouvent dans les études d'anthropologie médicale un terrain de réflexion privilégié.

Dans le premier cas, l'anthropologie est appliquée au domaine médical. Autrement dit, il s'agit d'utiliser l'anthropologie pour éclairer, par une connaissance des faits de culture, la pratique médicale. En témoignent un grand nombre de travaux contemporains, notamment américains, dont la finalité est une meilleure connaissance des facteurs culturels déterminant les comportements des malades, en vue d'accroître la rentabilité des programmes médicaux occidentaux auprès des populations concernées. Dans cette perspective, l'anthropologue travaille en conjonction avec le médecin auquel il apporte la contribution de sa méthode et de ses données, dans la mesure où les facteurs culturels ou ethniques peuvent aider à comprendre les causes, les caractéristiques ou les conséquences de la maladie, mais aussi des comportements des patients (par exemple : comprendre les raisons culturelles de la non observance).

Dans le second cas, la maladie est envisagée comme un domaine de l'anthropologie sociale. Cette tendance s'est affirmée en France avec Marc Augé. Celui-ci postule en effet que les pratiques relatives à la maladie sont indissociables d'un système symbolique articulé. Il a en particulier souligné l'étroite intrication entre les conceptions du désordre biologique et du désordre social, et a montré, en s'appuyant sur des études de cas en Côte d'Ivoire et au sud Togo, que les pratiques locales et les discours relatifs à la maladie relèvent de théories générales qui servent à penser (ou, plus exactement, qui ordonnent les symboles servant à penser) le social dans son ensemble.

Cette perspective théorique a ainsi conduit Marc Augé (1986) à récuser l'appellation "anthropologie médicale" dans la mesure où cette appellation suppose l'existence d'un champ constitué aux frontières définies, alors qu'il n'existe, dit-il, "qu'une anthropologie qui se donne des objets empiriques distincts", mais que ceux-ci constituent "un objet unique d'analyse". Il propose de lui substituer une "anthropologie de la maladie" au sens où la maladie n'est pour l'observateur qu'un objet d'occasion, qui doit permettre à l'étude anthropologique qui la prend pour objet d"'affiner ou (de) renouveler la problématique anthropologique". 
Le détour par l'étude des représentations de la maladie est, dans cette perspective, un itinéraire conseillé (voire un passage obligatoire) à l'anthropologue soucieux de cerner les systèmes de pensées et de conduites des individus, dans telle ou telle société. Comme l'écrit Augé, "Nombre d'ethnologues qui n'avaient pas la maladie pour objet premier de leur recherche, ont constaté qu'ils ne pouvaient pas essayer de comprendre la vie sociale, politique et religieuse des sociétés sans prendre en considération le système nosologique (l'élaboration du diagnostic, les prescriptions thérapeutiques, les institutions, les agents), bref la dimension sociale de la maladie" (1984). Toutefois, parler de dimension sociale de la maladie, ce n'est pas parler seulement de la cause sociale de la maladie, mais de 1"'armature intellectuelle" qui sert à penser la maladie.

Le but premier de l'anthropologie de la maladie est donc, non pas de concevoir des recherches finalisées sur une cible biomédicale, mais d'accéder par une autre porte à la connaissance de l'Homme en société.

Cette appellation n'a peut-être pas vraiment eu l'écho souhaité, puisque l'expression la plus utilisée aujourd'hui, non pas en France, mais sur la scène internationale, et notamment aux Etats-Unis où cette discipline est le plus développée, reste celle d'"anthropologie médicale", même lorsque c'est pour désigner des travaux réalisés dans l'optique définie par Marc Augé. Toutefois, cette approche a eu pour mérite de souligner que la maladie doit être envisagée comme un moyen, à l'instar d'autres domaines comme le religieux ou le politique par exemple, d'accéder à la compréhension et à la connaissance des sociétés.

\section{Remarques épistémologiques}

\section{$\underline{\text { Retour à la table des matières }}$}

De l'évolution qu'a connue ce domaine de recherche, il ressort que l'étude anthropologique de la maladie ne peut être abordée isolément. Ceci est vrai d'ailleurs non seulement pour les sociétés dites "traditionnelles", mais aussi pour les sociétés "modernes". Car bien évidemment, l'anthropologie de la maladie ne s'applique pas qu'aux sociétés dites exotiques, même si l'anthropologie médicale a longtemps négligé l'étude de nos propres sociétés et qu'elle ne s'est que récem- 
ment tournée vers elles. L'étude de la maladie renvoie à des interrogations qui sont universellement valides (le rapport au corps, le sentiment religieux, l'interaction entre individuel et social, etc.), et posent donc les mêmes questions, même si elles trouvent des réponses parfois différentes selon les sociétés ( $c f$. notamment Fainzang, 1989, pour une étude des modèles d'interprétation de la maladie en France, et des modèles d'accusations qui peuvent être formulées pour expliquer son apparition).

Cependant, faire de l'anthropologie de la maladie en Occident n'implique pas de chercher les survivances des pratiques traditionnelles dans la société moderne. On peut parfaitement s'intéresser aux pratiques les plus contemporaines, même les plus proches de celles que la médecine juge rationnelles.

À cet égard, la dichotomie entre "rationnel" et "magique" présente dans de nombreux travaux, et qui recouvre dans une certaine mesure l'usage parfois fait des notions de "moderne" et de "traditionnel", n'est sans doute pas la plus intéressante et empêche de voir que l'analyse sociale et symbolique peut parfaitement s'appliquer à des univers dits modernes et supposés rationnels. Or les anthropologues médicaux ont longtemps ignoré l'étude de nos sociétés, au motif qu'elles auraient évolué en dehors de l'emprise de la religion et des croyances si manifestes dans les médecines traditionnelles. Il y a donc un retard à rattraper, un vide à combler, à quoi s'attachent des auteurs contemporains comme Lock \& Gordon (1988).

Toutefois, un flou subsiste concernant les objectifs de ce champ de recherche, ce qui rend parfois complexes les relations entre les sciences sociales et les sciences médicales, et les conditions de la collaboration entre ces deux disciplines dans nos sociétés. Le problème se pose en effet de savoir si le dialogue entre médecine et anthropologie est celui qui existe entre deux sciences, puisque certains chercheurs tendent à considérer l'anthropologie comme une branche de la médecine, tandis que d'autres voient la médecine comme un champ de l'anthropologie. La question de savoir laquelle appartient à l'autre dans les travaux que nous réalisons en tant qu'anthropologues (dits "médicaux") est bien sûr celle de la finalité de notre science. En contrepoint de cette alternative, on peut se demander si l'on ne devrait pas plutôt considérer que la médecine occidentale est elle-même un objet pour l'anthropologie? Il faut accepter l'idée que la médecine n'est pas qu'un discours scientifique coopérant avec l'anthropologie face à la maladie (et face aux 
comportements des individus confrontés à la maladie), mais qu'elle peut être ellemême un objet pour l'anthropologie médicale, dans nos sociétés comme ailleurs. Un des paris de l'anthropologie médicale dans les sociétés occidentales est de se montrer capable de soumettre la biomédecine à ses outils analytiques, c'est-à-dire de considérer le système biomédical (son discours et ses pratiques) comme n'importe quelle pratique sociale profane ou comme n'importe quel objet exotique. D'abord parce qu'il n'est pas si sûr que les pratiques médicales occidentales soient si rationnelles qu'elles le revendiquent, ensuite parce que même les discours ou les conduites rationnelles peuvent faire l'objet d'une analyse anthropologique permettant de mettre au jour des phénomènes sociaux et culturels.

Parmi les questions couramment étudiées en anthropologie médicale, figure celle de l'efficacité. Il convient de remarquer toutefois que, là encore, articulée à la question de la rationalité, elle peut être un piège, car lorsqu'on s'interroge sur l'efficacité thérapeutique d'une cure dans une société donnée, on ne peut évacuer la dimension symbolique des traitements pour ne retenir que ce qui serait "rationnel", au regard des pratiques expérimentales occidentales. Si certains auteurs opposent éléments empiriques et éléments symboliques pour séparer ce qui est "efficace" du reste, cette séparation n'a pas de sens car la dimension symbolique, loin d'être un artefact inutile, est tout à fait essentielle. Comme l'a montré Lévi-Strauss (1962), il existe une nécessaire congruence entre le remède et le mal à soigner, conforme à une vision du monde, que l'anthropologue ne peut ignorer, puisqu'une de ses tâches est précisément de saisir les lois de la pensée symbolique. Dans tous les cas, il n'est pas du ressort ni de la compétence de l'anthropologue de se prononcer sur l'efficacité thérapeutique des médecines autres. La question de l'efficacité n'a d'intérêt que si elle est envisagée par rapport à la structure globale de la société, à l'ensemble de ses représentations et de ses institutions. C'est dire que, du point de vue anthropologique, ce qui importe est non pas de savoir si telle pratique est efficace ou non, mais pourquoi elle est pensée efficace par la société étudiée, quelle est sa signification et sa portée sociale, et comment est construite cette efficacité, ici comme ailleurs.

L'anthropologie, qui a appris à envisager la maladie autrement qu'avec une perspective bio-médicale sur les terrains exotiques, doit, de la même façon en Occident, considérer les matériaux ethnographiques sans prendre appui sur la perspective biomédicale. Par exemple, les débats complexes et houleux tenus dans 
le milieu des professionnels de la santé sur la question de savoir si le Sida est une maladie "contagieuse" ou "transmissible" n'est pas qu'une question purement technique. L'usage des notions de "contagion" ou de "transmission" est en soi un objet pour l'anthropologie puisque qu'il a des raisons et des implications sociales.

Par conséquent, ici comme ailleurs, des notions aussi évidentes que celles de "prévention" ou de "contagion" ne doivent, pas plus que celle d'"efficacité", être envisagées à l'aune de la perspective médicale, mais doivent toujours être réinterrogées et reconstruites par l'anthropologue.

Si appliquer au terrain occidental les problématiques forgées en terrain exotique (comme les théories de la contagion par exemple) permet d'examiner autrement l'ethnographie occidentale, il ne s'agit pas pour autant de transférer abusivement tous les concepts élaborés ailleurs. Car ils recouvrent une réalité sociale et culturelle spécifique et on ne peut pas les appliquer en toute liberté à des contextes nouveaux sans prendre le risque de les voir perdre de leur précision théorique. Il en va ainsi de mots comme ceux de "tribu" ou de "rituel", qu'il faut réexaminer avec beaucoup de précaution, sous peine de voir se diluer la précision de ces termes dans le contexte où ils ont été élaborés. Cela ne fait d'ailleurs parfois guère avancer la compréhension de ce que l'on étudie (parler de la "tribu" des médecins par exemple pour désigner ce groupe professionnel n'a pas de sens ni d'intérêt).

L'anthropologie médicale peut donc être utilement menée ici comme ailleurs, et de manière plus féconde encore, dans une perspective comparative. Si la comparaison est un aspect important de notre discipline en ce qu'elle est la condition même d'une pensée critique, le travail comparatif doit cependant être mené avec grande précaution. Car reconnaître la relativité de concepts et de catégories comme par exemple le normal et le pathologique - ne doit pas conduire au relativisme culturel. Il convient en effet d'envisager ces notions non pas comme figées, comme des constructions culturelles données de toute éternité, mais comme des constructions sociales, à l'intérieur d'une même société, sujettes aux variations des contextes sociaux dans lesquels elles ont été élaborées.

Pour conclure sur ce qui me paraît fondamental à retenir concernant ce domaine de recherche, l'anthropologie médicale (ou ce qu'il convient peut-être plus justement d'appeler l'"anthropologie de la maladie" si l'on s'accorde avec ses présupposés théoriques), n'est pas un domaine scientifique à part entière, mais c'est 
une pièce du puzzle qu'est l'ensemble du savoir anthropologique et dont elle doit aider à la reconstruction.

\section{RÉFÉRENCES BIBLIOGRAPHIQUES}

$\underline{\text { Retour à la table des matières }}$

ACKERKNECHT. E.H., 1946, "Natural Diseases and Rational Treatment in Primitive Medicine", Bulletin of the History of Medicine, 19: 467-497.

AUGE M., 1984, "Ordre biologique, ordre social : la maladie, forme élémentaire de 1'événement", in Le sens du mal : anthropologie, histoire, sociologie de la maladie, M. Augé et C. Herzlich (éd.), Paris, Editions des Archives Contemporaines, coll. Ordres Sociaux: 35-92.

AUGE M., 1986, "L'anthropologie de la maladie", L'Homme, 26 (1-2): 81-90.

BENOIST J. (éd.), 1996, Soigner au pluriel, Essais sur le pluralisme médical, Paris, Karthala.

BROQUA C, F. LOUX F, P. PRADO (éds.), 1998, "Sida: deuil, mémoire, nouveaux rituels", Ethnologie française, XXVIII, 1.

DOUGLAS M., 1992, Risk and Blame, Essays in Cultural Theory, London/New York: Routledge.

EVANS-PRITCHARD E. E., 1972, Sorcellerie, oracles et magie chez les Azande, Paris, Gallimard (Bibliothèque des Sciences Humaines).

FAINZANG S., 1986, L'intérieur des choses. Maladie, divination et reproduction sociale chez les Bisa du Burkina (préface de Marc Augé), Paris, l'Harmat$\tan$.

FAINZANG S., 1989, Pour une anthropologie de la maladie en France. Un regard africaniste. Paris, Éditions de l'École des Hautes Études en Sciences Sociales (collection: "Les Cahiers de l'Homme").

FAINZANG S., 1996, Ethnologie des anciens alcooliques. La liberté ou la mort. Paris, Presses Universitaires de France (coll. Ethnologies). 
FASSIN D., 1992, Pouvoir et maladie en Afrique, Paris, Presses Universitaires de France (coll.: "Les champs de la santé").

FOSTER, G. M., 1976, "Disease Etiologies in non-Western Medical Systems", American Anthropologist, 78 (4): 773-782.

HALLOWELL A. I., 1941, "The Social Function of Anxiety in a Primitive Society", American Sociological Review, 6: 869-881. Repris dans Culture, Disease and Healing, D. Landy, (ed.), New York, Macmillan, 1977.

HELMAN C., 2000, Culture, Health and Illness (4 ${ }^{\text {th }}$ ed), London Arnold.

HORTON R., 1967, "African Traditional Thought and Western Sciences, I \& II", Africa, 37, p.50-71 et 155-187.

KLEINMAN A., 1980, Patients and Healers in the Context of Culture, Berkeley, University of California Press.

LÉVI-STRAUSS Cl., 1958, "L'efficacité symbolique", in Anthropologie structurale I, Paris, Plon: 205-226.

LEVI-STRAUSS Cl., 1962, La pensée sauvage, Paris, Plon.

LOCK M. \& GORDON D. (eds.), 1988, Biomedecine Examined, Kluwer Academic Publishers, Dordrecht/Boston/London.

MASSE R., 1995, Culture et santé publique. Les contributions de l'anthropologie à la prévention et à la promotion de la santé, Montréal/Paris/Casablanca, Gaëtan Morin Editeur.

RIVERS W. H. R., 1924, Medicine, Magic and Religion, London, Kegan Paul.

TURNER V. W., 1972, Les tambours d'affliction: analyse des rituels chez les Ndembu de Zambie, Paris, Gallimard.

YOUNG A., 1976, "Some Implications of Medical Beliefs and Practices for Social Anthropology", in American Anthropologist, (78) 1: 5-24.

ZEMPLENI A., 1982, "Anciens et nouveaux usages sociaux de la maladie en Afrique", in Archives des Sciences Sociales des Religions, 54 (1): 5-19. 
ZEMPLENI A., 1985, "La maladie et ses causes: introduction", in Causes, origines et agents de la maladie chez les peuples sans écriture, I'Ethnographie, $n^{\circ}$ 96-97 : 13-44.

ZIMMERMANN F., 1995, Généalogie des médecines douces, Paris, Presses Universitaires de France.

\section{Fin du texte}

